\title{
Short communication: A comparison of cow cleanliness, fly population, and fly avoidance behaviors among docked, switch-trimmed, and switch-intact dairy cows in 3 commercial dairy herds
}

\author{
L. M. Frantz, ${ }^{1}$ E. A. Morabito, ${ }^{1}$ K. A. Dolecheck, ${ }^{1 *}$ and J. M. Bewley ${ }^{2}$ \\ ${ }^{1}$ Department of Animal and Food Sciences, University of Kentucky, Lexington 40546 \\ ${ }^{2}$ Alltech Inc., 3031 Catnip Hill Rd., Nicholasville, KY 40356
}

\section{ABSTRACT}

Tail docking is a controversial practice in the dairy industry. Proponents claim that tail docking keeps the udder cleaner, and therefore improves milk quality and decreases somatic cell count. Opponents of tail docking cite that it causes unnecessary pain, backed by multiple studies that demonstrate no positive benefits of tail docking and that tail docking increases aggravation from flies. The objective of this study was to evaluate and compare cow cleanliness, fly population, and fly-avoidance behaviors among cows with docked, switch-trimmed, and switch-intact tails. A total of 206 cows from 3 Kentucky dairy herds were included in the longitudinal observational study. Each farm included previously docked cows, switch-intact cows, and cows whose switches were trimmed at the initial farm visit. Researchers visited each farm every 2 wk for 8 wk to record cow cleanliness, teat cleanliness, fly population, and fly-avoidance behavior scores. No significant differences were found in cow cleanliness scores, teat cleanliness scores, fly population scores, skin twitching, or foot stomping counts among docked, switch-trimmed, or switch-intact cows. Although the fly population scores did not differ, the amount of tail swings among docked, switch-intact, and switch-trimmed cows were significantly different. The odds of exhibiting tail swinging were 2.63 times greater for docked cows than for switch-trimmed cows and 1.92 times greater than for switch-intact cows. Overall, switch trimming resulted in similar outcomes to tail docking, although neither showed improvements over intact tails.

Key words: tail docking, tail trimming, cow cleanliness, fly avoidance

Received April 13, 2018.

Accepted October 7, 2018.

*Corresponding author: karmella.dolecheck@uky.edu

\section{Short Communication}

Tail docking has been a common practice in the dairy industry since the 1980s (Barnett et al., 1999). Originally, tail docking was incorporated on farms because of assumed improvements in udder cleanliness, milk quality, and the comfort and health of milking personnel (Barnett et al., 1999; Eicher et al., 2000; Stull et al., 2002). However, both Stull et al. (2002) and Sutherland and Tucker (2011) concluded in their reviews of the literature that there was no scientific evidence for these supposed benefits. Additionally, the American Veterinary Medical Association opposes tail docking, stating that docking provides no benefit to the animal and can lead to distress (AVMA, 2017). Distress from tail docking includes both acute stress at the time of docking (Tom et al., 2002) and increased stress for the animals during fly season because of their inability to remove flies (Eicher and Dailey, 2002; AVMA, 2017). Eicher et al. (2001) found that docked cows had a greater number of flies on them and showed a greater number of flyavoidance behaviors. This can especially be a problem if biting flies are present, as they have been linked to disrupted grazing, slower growth, reduced milk production, and decreased weight gain (Eicher and Dailey, 2002).

Regardless of the lack of scientific evidence supporting the practice, 2008 and 2010 surveys of US dairy farms found that most dairies (82 and 69\%, respectively) continued to practice tail docking (Fulwider et al., 2008; Lombard et al., 2010). Because of increased consumer concerns about the welfare implications of tail docking, the National Milk Producers Federation Board of Directors passed a tail docking ban in the United States that went into effect on January 1, 2017, for all producers enrolled in the Farmers Assuring Responsible Management (FARM) Animal Care Program (NMPF, 2015). The FARM program encompasses more than $90 \%$ of the US milk supply, making the tail docking ban relevant to most farms in the United States. Producers affected by this ban may seek alternative 
strategies to manage tail length. One proposed alternative method is to trim the long hair off the end of the tail (i.e., switch trimming; Stull et al., 2002). Recently, Ingle et al. (2018) compared bacterial counts and udder cleanliness scores between switch-trimmed and switchintact cows, finding no statistical difference. Those authors suggested that further research was needed to determine how switch trimming affected fly counts. The objective of our study was to evaluate and compare cow cleanliness, fly population, and fly-avoidance behaviors among cows with docked, switch-trimmed, and switchintact tails. We hypothesized that cleanliness scores would not be different between the 3 treatments. Additionally, we hypothesized that fly population scores and fly-avoidance behaviors would not be different between switch-intact and switch-trimmed tails, but that cows with switch-intact and switch-trimmed tails would have lower fly population scores and show fewer fly-avoidance behaviors than cows with docked tails. The study was conducted across multiple herds over an 8-wk period to determine if results were consistent across different herd scenarios and if differences remained over time.

This study was conducted between October 9 and December 4, 2013, using 206 lactating dairy cows from 3 Kentucky dairy herds. Each farm used different housing and management systems. Cows from farm A $(\mathrm{n}=31)$ were housed in a tiestall barn with rubber mattresses and milked 2 times per day. Cows from farm B ( $\mathrm{n}=$ 109) were housed in a freestall barn with mattresses and milked 2 times per day. Cows from farm C ( $\mathrm{n}=$ 66) were housed in a compost-bedded pack barn and milked 3 times per day.

No cows were docked specifically for our study, but all farms contained previously docked cows. All remaining cows with intact tails and even-numbered ear tags had their tails trimmed with a circular, drill-powered trimmer (TailWell Power Tail Trimmer, Shoof International Ltd., Cambridge, New Zealand). The trimmer was attached to a hand drill and the end of the cows' tail was inserted into the trimmer opening. The hair was removed by turning on the drill and sliding it up to the base of the tail in one motion. The remaining cows (intact tails and odd numbered ear tags) served as the control group and their switches remained untrimmed. Cows in the trimmed group were trimmed during the initial farm visit (October 9, 2013) but were not trimmed at any of the other farm visits that took place every other week for the remainder of the study $(\mathrm{n}=4$; Oct. 23 , Nov. 6, Nov. 20, and Dec. 4). At each visit after the initial visit, data collection included cow cleanliness scores, teat cleanliness scores, fly population scores, and fly-avoidance behavior scores. Because the scoring systems used in the current study were subjec- tive, observer bias is possible. An attempt was made to minimize this bias by using only a single, previously trained observer for all observations. The individual completed all data collection by traveling between farms in the same day.

Initial cow and teat cleanliness scoring occurred during milking at the first farm visit while the cows' tails were being trimmed. During the remaining visits, cows were scored in either the milking parlor or the housing area, depending on whether milking was occurring at the time of the visit. Cow cleanliness was evaluated by assigning a score of 0 to 3 for each of the flank, leg, and udder areas $(0=$ no debris, $1=$ flecks of debris, $2=$ a film or thicker chunks of debris, $3=$ thick caking of debris; Tucker et al., 2001). Teat cleanliness was evaluated for each individual teat using a scoring system ranging from 0 to 4 , where $0=$ clean (no visible dirt), $1=$ almost clean $(<10 \%$ of the area dirty $), 2=$ slightly dirty $(10-20 \%$ of the area dirty), $3=$ dirty $(20-50 \%$ of the area dirty), and $4=$ extremely dirty ( $>50 \%$ of the area dirty; Hovinen et al., 2005).

Fly population scores and fly-avoidance behaviors were not recorded during the initial farm visit. During the remaining visits, fly population was observed for $1 \mathrm{~min}$ per cow and categorized as 0 (no flies), 1 (less than 5 flies), 2 ( 5 to 15 flies), or 3 (15 or more flies). After fly population scores were assigned, the occurrence of each fly avoidance behavior was recorded for each cow during a 2-min observation period; this length of time was chosen to allow enough time for all data collection within $1 \mathrm{~d}$. The herds were all visited in the same order on each visit day. Although fly populations may have differed between herds based on time of day, the herd visits were comparable to each other and all tail statuses within a herd were comparable. Avoidance behaviors included foot stomping (raising and lowering of a foot in 1 spot), skin twitching (rippling of skin), and tail swinging (movement of tail greater than $45^{\circ}$ from vertical; Eicher and Dailey, 2002).

The MIXED procedure of SAS (SAS Institute Inc., Cary, NC) was used to evaluate the effects of tail status (docked, switch trimmed, or switch intact) on udder, flank, leg, and individual teat cleanliness scores. Within all models, sample date (visit number $0,1,2,3$, or 4 ), herd (A, B, or $\mathrm{C}$ ), and all 2-way interactions (sample date $\times$ herd, sample date $\times$ tail status, and tail status $\times$ herd) were included as fixed effects. These covariates were included to determine if differences between tail statuses would change between herds or across time (sample date). Within models, cow was included as a random effect and cow within herd was considered as the repeated subject across sample dates. Stepwise backward elimination was used to remove nonsignifi- 
cant interactions one at a time (starting with the least significant interaction) until all interaction $P$-values were less than 0.05 .

The model for fly population score was identical to the previously described MIXED model but did not include sample date 0 because no measurements were taken at that time. A similar MIXED model (not including sample date 0) was also used to evaluate skin twitching and foot stomping, but fly population score $(0,1,2$, or 3$)$ was additionally included as a covariate. No interactions among fly population score and other covariates were included in the skin twitching or foot stomping models.

No tail swings were observed in most of the tail swing observations; therefore, tail swing observations were categorized as either 0 (no tail swings) or 1 ( $\geq 1$ tail swing). The GENMOD procedure was used to evaluate the effects of tail status (docked, switch trimmed, or switch intact) on the categorical tail swing variable. Sample date (visit number 1, 2, 3, or 4), herd (A, B, or $\mathrm{C})$, and fly population score $(0,1,2$, or 3$)$ were included in the model as fixed effects. Again, cow was included as a random effect and cow within herd was considered as the repeated subject across sample dates, but no interactions were included in the model. Significance was defined as $P<0.05$.

Cleanliness scores for the udder, flank, leg, and each individual teat by tail status group (docked, switch trimmed, or switch intact) are presented in Table 1. All hygiene scores were relatively low $(<2$ on 0 to 3 or 0 to 4 scales), possibly because the study was conducted during colder, drier months when cleanliness is generally less of an issue. Schreiner and Ruegg (2003) found that cows with udder hygiene scores $>2$ were more likely to have major pathogens isolated from milk samples.

Tail status did not have a significant effect on any of the 7 different cleanliness scores $(P \geq 0.05)$. Both
Stull et al. (2002) and Schreiner and Ruegg (2002) also found that docking did not improve cleanliness of the udder and body compared with intact tails, whereas Lombard et al. (2010) found that tail docking was associated with decreased cleanliness. Ours is one of the first studies considering how switch trimming affected cow cleanliness. Our results are in agreement with Ingle et al. (2018), who found no effect of switch trimming on udder and teat hygiene. Additionally, we found no differences in flank or leg hygiene associated with switch trimming. Overall, switch trimming appeared to perform similarly to both tail docking and intact tails regarding all measures of hygiene. Udder, flank, and leg hygiene were all affected by the interaction of herd and sample date $(P<0.01)$. Eicher et al. $(2001)$ noted that cleanliness scores can vary because of weather, housing types, water consumption, ration, and frequency of cleaning; however, evaluating those variables was beyond the objective of our study, so conclusions regarding their contribution to our results cannot be made.

Fly population scores, skin twitching, and foot stomping results by tail status group are presented in Table 2. Fly population scores were generally low $(<1$, indicating fewer than 5 flies observed within a 1-min period), resulting in low occurrences of fly-avoidance behaviors. This may have resulted from the study being conducted in cooler months, when fly populations are generally lower. Further experiments during seasons with greater fly populations should be conducted to confirm our findings.

Different from previous studies, we found no significant differences in fly population scores among the 3 tail statuses. Both Phipps et al. (1995) and Eicher and Dailey (2002) found that the number of flies was greater on docked cows compared with switch intact cows. In fact, Eicher and Dailey (2002) found twice as many flies on docked cows' rumps. The lack of difference found in our study could have occurred because

Table 1. Mean \pm SD udder, flank, leg, and teat cleanliness scores by tail status ${ }^{1}$

\begin{tabular}{|c|c|c|c|c|c|c|}
\hline \multirow[b]{2}{*}{ Item } & \multicolumn{3}{|c|}{ Tail status (no.) } & \multicolumn{2}{|c|}{ Degrees of freedom } & \multirow[b]{2}{*}{$P$-value } \\
\hline & Docked & Switch trimmed & Switch intact & Numerator & Denominator & \\
\hline Flank & $1.34 \pm 0.03(91)$ & $1.31 \pm 0.04$ & $1.36 \pm 0.04(63)$ & 2 & 807 & 0.68 \\
\hline Leg & $1.87 \pm 0.04(90)$ & $1.89 \pm 0.05(53)$ & $1.82 \pm 0.04(61)$ & 2 & 767 & 0.45 \\
\hline Left front teat & $0.83 \pm 0.05(91)$ & $0.84 \pm 0.06(53)$ & $0.80 \pm 0.05(62)$ & 2 & 812 & 0.83 \\
\hline Right front teat & $0.70 \pm 0.05$ & $0.84 \pm 0.06$ & $0.79 \pm 0.06(62)$ & 2 & 820 & 0.17 \\
\hline
\end{tabular}

${ }^{1}$ Udder, flank, and leg cleanliness were scored from 0 to 3 , where $0=$ no debris, $1=$ flecks of debris, $2=$ a film or thicker chunks of debris, $3=$ thick caking of debris (Tucker et al., 2001). Teat cleanliness was scored from 0 to 4 , where $0=$ clean (no visible dirt), $1=$ almost clean $(<10 \%$ of the area dirty), $2=$ slightly dirty (10 to $20 \%$ of the area dirty), $3=$ dirty (20 to $50 \%$ of the area dirty), and $4=$ extremely dirty ( $>50 \%$ of the area dirty; Hovinen et al., 2005). 
Table 2. Mean \pm SD fly population score, foot stomping rate, and skin twitching rate by tail status ${ }^{1}$

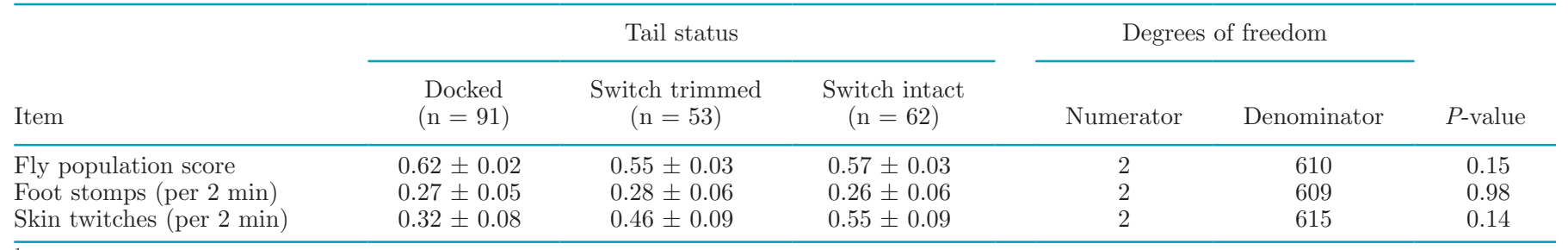

${ }^{1}$ Fly population was observed for 1 min per cow and categorized as 0 (no flies), 1 (less than 5 flies), 2 ( 5 to 15 flies), or 3 (15 or more flies). The incidence of each avoidance behavior was recorded for each cow during a 2-min observation period. Foot stomping was considered when a cow raised and lowered a foot in the same spot and skin twitching was a rippling of the skin (Eicher and Dailey, 2002).

fewer flies were present in general during the season the study was conducted (i.e., late fall). The interaction of herd and sample date affected fly populations scores. All herds experienced a general decrease in fly population scores over time, likely following decreased temperatures. Similar to hygiene scores, fly population score differences among herds could be attributed to different herd management practices, housing types, and weather conditions experienced. However, we did not evaluate these factors, so conclusions on why differences between herds and sample dates existed cannot be made.

As expected, as fly population score increased, skin twitching, foot stomping, and tail swinging increased to combat the increase in flies $(P<0.01)$. Skin twitching did not differ by tail status, sample date, or herd. Foot stomping did not differ by tail status but was affected by the interaction between sample date and herd $(P<$ $0.01)$. Tail swinging differed by sample date and tail status $(P<0.01)$. The odds of docked cows showing signs of tail swinging (tail swing category 1 ) were 2.63 times greater than switch trimmed cows and 1.92 times greater than switch intact cows. Tail swinging did not differ between switch-trimmed cows and switch-intact cows. We observed no difference in fly population between cows with different tail statuses; therefore, the fact that docked cows were swinging their tails more could indicate some level of discomfort. Similarly, Eicher et al. (2006) found that docked heifers showed increased sensitivity to heat and cold compared with undocked heifers, similar to the reaction to phantom limb pain found in human amputees.

Overall, docked, switch-trimmed, and switch-intact dairy cows displayed similar hygiene scores, fly population scores, foot stomping behavior, and skin twitching behavior. The only difference found between tail statuses was an increased odds of tail swinging in docked versus switch-intact or switch-trimmed cows. The results of our study confirm previous findings that tail trimming has no added hygiene benefits compared with intact tails and adds valuable information on the effects of tail trimming on fly population and fly-avoidance behaviors. Farmers seeking alternative tail management methods should not expect hygiene or fly count improvements when trimming tails and should keep this in mind when considering the cost of labor and supplies to conduct regular switch trimming in their herd.

\section{ACKNOWLEDGMENTS}

The authors thank Shoof International Ltd. (Cambridge, New Zealand), who provided financial support for this study. We also appreciate the 3 dairy producers who provided us access to their herds to complete this research. Finally, we thank Derek Nolan (University of Kentucky, Lexington) for his assistance completing this project.

\section{REFERENCES}

AVMA. 2017. AVMA Policies: Tail Docking of Cattle. Accessed Oct. 24, 2017. https://www.avma.org/KB/Policies/Pages/Tail-Docking -of-Cattle.aspx.

Barnett, J. L., G. Coleman, P. Hemsworth, E. Newman, S. FewingsHall, and C. Ziini. 1999. Tail docking and beliefs about the practice in the Victorian dairy industry. Aust. Vet. J. 77:742-747.

Eicher, S. D., and J. Dailey. 2002. Indicators of acute pain and fly avoidance behaviors in Holstein calves following tail-docking. J. Dairy Sci. 85:2850-2858.

Eicher, S. D., J. Morrow-Tesch, J. Albright, J. Dailey, C. Young, and L. Stanker. 2000. Tail-docking influences on behavioral, immunological, and endocrine responses in dairy heifers. J. Dairy Sci. 83:1456-1462.

Eicher, S. D., J. Morrow-Tesch, J. Albright, and R. Williams. 2001. Tail-docking alters fly numbers, fly-avoidance behaviors, and cleanliness, but not physiological measures. J. Dairy Sci. 84:1822-1828.

Eicher, S. D., H. W. Cheng, A. D. Sorrells, and M. M. Schutz. 2006. Short communication: Behavioral and physiological indicators of sensitivity or chronic pain following tail docking. J. Dairy Sci. 89:3047-3051.

Fulwider, W. K., T. Grandin, B. Rollin, T. Engle, N. Dalsted, and W. Lamm. 2008. Survey of dairy management practices on one hundred thirteen north central and northeastern United States dairies. J. Dairy Sci. 91:1686-1692.

Hovinen, M., A.-M. Aisla, and S. Pyörälä. 2005. Visual detection of technical success and effectiveness of teat cleaning in two automatic milking systems. J. Dairy Sci. 88:3354-3362.

Ingle, H. D., C. A. Rice, R. A. Black, S. Z. Childers, N. L. Eberhart, M. E. Prado, and P. D. Krawczel. 2018. Effect of switch trimming 
on udder and teat hygiene of dairy cows. J. Appl. Anim. Welf. Sci. $21: 239-243$.

Lombard, J. E., C. Tucker, M. Von Keyserlingk, C. Kopral, and D. Weary. 2010. Associations between cow hygiene, hock injuries, and free stall usage on US dairy farms. J. Dairy Sci. 93:4668-4676.

NMPF. 2015. NMPF Board Advances Phase-Out of Tail Docking. National Milk Producers Federation. Accessed Aug. 21, 2017. http:/ /www.nmpf.org/latest-news/press-releases/oct-2015/nmpf-board -advances-phase-out-tail-docking.

Phipps, A., L. Matthews, and G. Verkerk. 1995. Tail docked dairy cattle: Fly induced behaviour and adrenal responsiveness to ACTH. Page 61 in Proc. of the New Zealand Society of Animal Production. New Zealand Society of Animal Production, Cambridge, New Zealand.

Schreiner, D. A., and P. Ruegg. 2002. Effects of tail docking on milk quality and cow cleanliness. J. Dairy Sci. 85:2503-2511.
Schreiner, D. A., and P. Ruegg. 2003. Relationship between udder and leg hygiene scores and subclinical mastitis. J. Dairy Sci. 86:34603465.

Stull, C. L., M. A. Payne, S. L. Berry, and P. J. Hullinger. 2002. Evaluation of the scientific justification for tail docking in dairy cattle. J. Am. Vet. Med. Assoc. 220:1298-1303.

Sutherland, M. A., and C. B. Tucker. 2011. The long and short of it: A review of tail docking in farm animals. Appl. Anim. Behav. Sci 135:179-191.

Tom, E., J. Rushen, I. Duncan, and A. De Passillé. 2002. Behavioural, health and cortisol responses of young calves to tail docking using a rubber ring or docking iron. Can. J. Anim. Sci. 82:1-9.

Tucker, C. B., D. Fraser, and D. M. Weary. 2001. Tail docking dairy cattle: Effects on cow cleanliness and udder health. J. Dairy Sci. 84:84-87. 